\title{
In vitro emergence of carbapenem resistance in extended-spectrum $\beta$ - lactamase-producing Klebsiella pneumoniae clinical isolates
}

\author{
Hugo Edgardo Villar ${ }^{1}$, Gabriela Santana², Monica Beatriz Jugo ${ }^{1}$, Patricia Perren², Mariana Hidalgo ${ }^{1}$, \\ Patricia Alonso ${ }^{2}$, Gustavo Maccallini ${ }^{1}$ \\ ${ }^{1}$ Department of Clinical Bacteriology, Laboratorio Hidalgo, Buenos Aires, Argentina \\ ${ }^{2}$ Department of Clinical Bacteriology, Sanatorio San Lucas, Buenos Aires, Argentina
}

Key words: Ertapenem; ESBL; carbapenem; resistance; Klebsiella pneumoniae.

J Infect Dev Ctries 2015; 9(2):218-221. doi:10.3855/jidc.5563

(Received 12 July 2014 - Accepted 07 August 2014)

Copyright (C) 2015 Villar et al. This is an open-access article distributed under the Creative Commons Attribution License, which permits unrestricted use, distribution, and reproduction in any medium, provided the original work is properly cited.

Klebsiella pneumoniae is thought to be the most common species producing ESBLs, and almost $50 \%$ of Klebsiella pneumoniae isolates produce ESBL in some countries [1]. Furthermore, antimicrobial co-resistance within ESBL-producing isolate communities limits the number of drugs that are effective against these strains, leaving carbapenems as the most reliable agents [2-3].

Carbapenem resistance can arise through the production of acquired metallo- $\beta$-lactamases such as VIM and IMP or from production of non-metallocarbapenemases of the IMI/NMC, SME, OXA, or KPC families. Resistance may also be due to a combination of impermeability caused by porin loss and ESBL or AmpC $\beta$-lactamase production. This impermeability was documented in several cases in which carbapenem-resistant $K$. pneumoniae emerged in vivo in response to ertapenem [4-5], meropenem [67], and less frequently to imipenem exposure [8].

Among the several factors that contribute to the appearance and spread of acquired antibiotic resistance, the selection of high-level resistant mutants is especially important. In the current study, we examined a collection of ESBL-producing $K$. pneumoniae with the aim of evaluating the ability of carbapenem exposure to select single-step resistant mutants.

Clinical $K$. pneumoniae isolates $(\mathrm{n}=35)$ were obtained from inpatients admitted to the Sanatorio San Lucas, Buenos Aires, Argentina $(\mathrm{n}=18)$ and adult male outpatients $(\mathrm{n}=17)$ who attended the Laboratorio Hidalgo, Buenos Aires, Argentina.
The API 20 E system (bioMerieux, Marcy L'Étoile, France) was used for biochemical identification of all strains. Antibiotic minimal inhibitory concentrations (MICs) were determined by the epsilometric test (Etest; bioMerieux) and data was interpreted in accordance with Clinical and Laboratory Standards Institute (CLSI) guidelines [9]. Susceptibility to cefoxitin was determined by disc diffusion.

For phenotypic detection of ESBL, an overnight culture suspension of the test isolate, adjusted to 0.5 McFarland's standard, was inoculated onto the surface of a Mueller-Hinton agar plate. Cefotaxime $(30 \mu \mathrm{g})$ and cefotaxime-clavulanic acid $(30 \mu \mathrm{g} / 10 \mu \mathrm{g})$ discs were placed $20 \mathrm{~mm}$ apart on the agar. Similarly, ceftazidime $(30 \mu \mathrm{g})$ and ceftazidime-clavulanic acid $(30 \mu \mathrm{g} / 10 \mu \mathrm{g})$ discs were also placed $20 \mathrm{~mm}$ apart. An increase of $\geq 5 \mathrm{~mm}$ in the zone diameter for an antimicrobial agent tested in combination with clavulanic acid versus the zone when tested alone was considered positive for ESBL production. AmpC $\beta$ lactamases were phenotypically detected using inhibitor-based assays with cefoxitin discs $(30 \mu \mathrm{g})$ and boronic acid $(300 \mu \mathrm{g})$ (Laboratorios Britania, Buenos Aires, Argentina).

The modified Hodge test (MHT) was performed as described previously, with a $10-\mu \mathrm{g}$ imipenem disk [9].

The frequency of spontaneous single-step mutation was determined by spreading cultures $\left(\sim 10^{7}-10^{9}\right.$ $\mathrm{cfu} / \mathrm{ml})$ in $100 \mu \mathrm{l}$ saline onto multiple Mueller-Hinton agar plates, each containing $0.5 \mu \mathrm{g} / \mathrm{ml}$ of a single carbapenem (ertapenem, meropenem, or imipenem). 
Plates were incubated aerobically at $35^{\circ} \mathrm{C}$ for $48-72$ hours. The mutation frequency was calculated as the number of resistant colonies per inoculum. For each isolate, a representative mutant that was stable after three subcultures was conserved for further susceptibility testing.

Clinical $K$. pneumoniae isolates $(\mathrm{n}=35)$ were subjected to antibiotic testing. All the clinical strains were susceptible to ertapenem, meropenem, and imipenem, according to their CLSI breakpoints. MHTs were negative, indicating the lack of carbapenemase production. Three strains were found to be resistant to cefoxitin by disc diffusion tests, but were negative in synergism tests with boronic acid, indicating the absence of AmpC $\beta$-lactamases. All the isolates displayed synergistic activity between third-generation cephalosporins and clavulanic acid, and thus were phenotypically characterized as ESBL producers. The thirty-five $K$. pneumoniae strains were tested for incidence of single-step mutation by exposure to 0.5 $\mu \mathrm{g} / \mathrm{ml}$ ertapenem, meropenem, or imipenem. Singlestep mutants were isolated from 16 strains (45.7\%) exposed to ertapenem and 6 strains $(17.1 \%)$ exposed to meropenem, but no mutants were selected with imipenem exposure. The single-step mutation frequencies were $6.7 \times 10^{-7}-1.6 \times 10^{-9}$ with ertapenem and $5.4 \times 10^{-7}-1.9 \times 10^{-10}$ with meropenem. One representative stable mutant derived from each isolate was selected for further testing. The characteristics of the ertapenem-selected mutants are displayed in Table 1. When compared with parental strains, the MICs for the ertapenem-selected mutants increased 5.3-fold (LH8) to 500-fold (LH16), 2.7-fold (LH4) to 125-fold (LH3) for meropenem, and no increase (LH11) to 16fold (LH12) for imipenem. Cross-resistance (MIC >1 $\mu \mathrm{g} / \mathrm{ml}$ ) was observed to both meropenem and imipenem in four strains (LH3 EM, LH6 EM, LH9 $\mathrm{EM}$, and LH12 EM) and to meropenem alone in two strains (LH14 EM and LH16 EM).

The characteristics of the meropenem-selected mutants are displayed in Table 2. When compared with parental strains, the MICs for the meropenemselected mutants increased 64-fold (LH6) to 375-fold (LH7 and LH16) for ertapenem, 16-fold (LH9) to 64fold (LH8) for meropenem, and no increase (LH7) to 8-fold (LH8) for imipenem. Cross-resistance to ertapenem was detected in all mutants and to imipenem in two mutants (LH8 MM and LH9 MM).

Mutant $K$. pneumoniae isolates that were resistant to carbapenem antibiotics were generated in this study, demonstrating that resistance against ertapenem and meropenem can emerge in clinical ESBL-producing $K$. pneumonia isolates. In addition, several of the mutants selected with ertapenem or meropenem displayed cross-resistance to imipenem. Previous research indicates that ESBL expression in combination with the loss of porin expression can reduce susceptibility to carbapenems in clinical K. pneumoniae and E. coli isolates [10-12]. Whilst numerous outbreaks of carbapenem-resistant $K$. pneumoniae possessing various carbapenemases have been documented; an outbreak caused by an ertapenem-resistant, CTX-M15-producing clonal $K$. pneumoniae strain expressing an OmpK36 porin variant was only recently described [13-14].

The mutants selected as a result of exposure to ertapenem or meropenem exhibited dramatically increased MICs when challenged with either of the two antibiotics, indicating that the mechanisms of uptake are likely to be similar. No mutants were selected when imipenem was used, but some of the ertapenem- and meropenem-selected mutants nevertheless displayed cross-resistance to imipenem. Despite this observation, most of the mutants remained susceptible to imipenem. This suggests that porin loss is more significant for ertapenem resistance and meroperem resistance than for imipenem resistance [15]. The current study has some limitations. For example, the selection was performed using a stable carbapenem concentration $(0.5 \mu \mathrm{g} / \mathrm{ml})$ and not related to carbapenem MICs. It was not possible to perform molecular characterization of the relevant bacterially expressed enzymes, to detect isolates expressing multiple enzymes, or to assess outer membrane permeability patterns. Future studies may allow the resistance mechanisms to be elucidated.

It is important to note that single-step mutants showing resistance to ertapenem and meropenem were selected in vitro with relative ease. This is of clinical importance because such resistance could inadvertently make subsequent therapy using imipenem less effective or even ineffective.

This variety of in vitro resistant mutants may reflect the growing number of studies describing treatment failure with ertapenem [4-5] and meropenem [6-7]. Additionally, nosocomial outbreaks by carbapenem-resistant strains were recently documented in which resistance was not mediated by carbapenemases [13-14]. 
Table 1. Minimal inhibitory concentrations of ertapenem, meropenem, and imipenem against $K$ pneumoniae isolates and the corresponding mutants selected with ertapenem.

\begin{tabular}{|c|c|c|c|}
\hline \multirow[b]{2}{*}{ Isolate/Mutant } & \multicolumn{3}{|c|}{ Minimal inhibitory concentration (mg/l) } \\
\hline & Ertapenem & Meropenem & Imipenem \\
\hline LH1 P & 0.047 & 0.032 & 0.19 \\
\hline LH1 EM & 1.5 & 0.25 & 0.25 \\
\hline LH2 EM & 2 & 0.75 & 0.38 \\
\hline LH3 P & 0.25 & 0.064 & 0.19 \\
\hline LH3 EM & $>32$ & 8 & 2 \\
\hline LH4 EM & 2 & 0.25 & 0.25 \\
\hline LH5 P & 0.064 & 0.094 & 0.25 \\
\hline LH5 EM & 4 & 0.5 & 0.38 \\
\hline LH6 P & 0.25 & 0.125 & 0.38 \\
\hline LH6 EM & 16 & 2 & 2 \\
\hline LH7 P & 0.064 & 0.094 & 0.25 \\
\hline LH9 P & 0.19 & 0.125 & 0.38 \\
\hline LH9 EM & $>32$ & 2 & 2 \\
\hline LH10 P & 0.016 & 0.064 & 0.25 \\
\hline LH10 EM & 1 & 0.25 & 0.25 \\
\hline LH11 P & 0.032 & 0.047 & 0.25 \\
\hline LH11 EM & 2 & 0.25 & 0.25 \\
\hline LH12 P & 0.25 & 0.125 & 0.25 \\
\hline LH12 EM & $>32$ & 8 & 4 \\
\hline LH13 P & 0.125 & 0.064 & 0.19 \\
\hline LH13 EM & 2 & 0.5 & 0.25 \\
\hline LH14 P & 0.25 & 0.064 & 0.38 \\
\hline
\end{tabular}

\footnotetext{
P: parent strain, EM: mutant selected with ertapenem;
}

Table 2. Minimal inhibitory concentrations of ertapenem, meropenem, and imipenem against $K$ pneumoniae isolates and the corresponding mutants selected with meropenem

\begin{tabular}{cccc}
\hline & & Minimal inhibitory concentration (mg/l) & Imipenem \\
\cline { 2 - 4 } Isolate/Mutant & Ertapenem & Meropenem & 0.19 \\
LH3 P & 0.25 & 0.064 & 0.25 \\
LH3 MM & $>32$ & 4 & 0.25 \\
LH6 P & 0.25 & 0.125 & 0.5 \\
LH6 MM & 16 & 2 & 0.25 \\
LH7 P & 0.064 & 0.094 & 0.25 \\
LH7 MM & 24 & 4 & 0.25 \\
LH8 P & 0.19 & 0.125 & 2 \\
LH8 MM & $>32$ & 8 & 0.38 \\
LH9 P & 0.19 & 0.125 & 2 \\
LH9 MM & 16 & 2 & 0.19 \\
LH16 P & 0.032 & 0.047 & 2 \\
LH16 MM & 12 & 2 & 0.38 \\
\hline
\end{tabular}

P: parent strain, MM: mutant selected with meropenem 
In conclusion, this study demonstrates the rapid acquisition of decreased carbapenem susceptibility in ESBL-producing $K$. pneumoniae clinical isolates. The use of ertapenem in high-inoculum infections or in undrained infection foci should therefore be monitored to reduce the risk of resistance selection.

\section{References}

1. Paterson DL, Ko WC, Von Gottberg A, Mohapatra S, Casellas JM, Goossens H, Mulazimoglu L, Trenholme G, Klugman KP, Bonomo RA, Rice LB, Wagener MM, McCormack JG, Yu VL (2014) Antibiotic Therapy for Klebsiella pneumoniae Bacteremia: Implications of Production of Extended-Spectrum $\beta$-Lactamases. Clin Infect Dis 39: 31-37.

2. Schwaber MJ, Navon-Venezia S, Schwartz D, Carmeli Y (2005) High levels of antimicrobial coresistance among extended-spectrum $\quad \beta$-lactamase producing Enterobacteriaceae. Antimicrob Agent Chemother 49:2137139.

3. Tumbarello M, Sanguinetti M, Montuori E, Trecarichi EM, Posteraro B,Fiori B, Citton R, D'Inzeo T, Fadda G, Cauda R, Spanu T (2007) Predictors of mortality in blood stream infections caused by extended-spectrum $\beta$-lactamaseproducing Enterobacteriaceae: importance of inadequate initial antimicrobial treatment. Antimicrob Agents Chemother 51: 1987-1994.

4. Elliott E, Brink AJ, van Greune J, Els Z, Woodford N, Turton J, Warner M, Livermore DM (2006) In vivo development of ertapenem resistance in a patient with pneumonia caused by Klebsiella pneumoniae with an extended-spectrum betalactamase. Clin Infect Dis 42: 95-98.

5. Skurnik D, Lasocki S, Bremont S, Muller-Serieys C, Kitzis MD, Courvalin P , Andremont A, Montravers P (2010) Development of ertapenem resistance in a patient with mediastinitis caused by Klebsiella pneumoniae producing an extended-spectrum beta-lactamase. J Med Microbiol 59: 115119.

6. Findlay J, Hamouda A, Dancer SJ, Amyes SG (2012) Rapid acquisition of decreased carbapenem susceptibility in a strain of Klebsiella pneumoniae arising during meropenem therapy. Clin Microbiol Infect 18: 140-146.

7. Ruiz E, Ocampo-Sosa AA, Rezusta A, Revillo MJ, Román E, Torres C, Martinez-Martinez L (2012) Acquisition of carbapenem resistance in multiresistant Klebsiella pneumoniae strains harbouring blaCTX-M-15, qnrS1 and aac(6')-Ib-cr genes. J Med Microbiol 61: 672-677.

8. Cuzon G, Naas T, Guibert M, Nordmann P (2010) In vivo selection of imipenem-resistant Klebsiella pneumoniae producing extended-spectrum beta-lactamase CTX-M-15 and plasmid-encoded DHA-1 cephalosporinase. Int J Antimicrob Agents 35: 265-268.

9. Clinical and Laboratory Standards Institute (2013) Performance standards for antimicrobial susceptibility testing. Twenty-third informational supplement, M100-S 23. Wayne (PA), Clinical and Laboratory Standards Institute.

10. Girlich D, Poirel L, Nordmann P (2009) CTX-M expression and selection of ertapenem resistance in Klebsiella pneumoniae and Escherichia coli. Antimicrob Agents Chemother 53: 832-834.

11. Wang XD, Cai JC, Zhou HW, Zhang R, Chen GX (2009) Reduced susceptibility to carbapenems in Klebsiella pneumoniae clinical isolates associated with plasmidmediated $\beta$-lactamases production and OmpK36 porin deficiency. J Med Microbiol 58: 1196-1202.

12. Villar HE, Jugo MB, Visser M, Hidalgo M, Hidalgo G, Maccallini GC (2014) Rápida adquisición de resistencia in vitro al ertapenem en Escherichia coli productora de beta lactamasa de espectro extendido. Rev Esp Quimioter 27: 5155.

13. Novais A, Rodrigues C, Branquinho R, Antunes P, Grosso F, Boaventura L, Ribeiro G, Peixe L (2012) Spread of an OmpK36-modified ST15 Klebsiella pneumoniae variant during an outbreak involving multiple carbapenem-resistant Enterobacteriaceae species and clones. Eur J Clin Microbiol Infect Dis 31:3057-3063.

14. Poulou A, Voulgari E, Vrioni G, Koumaki V, Xidopoulos G, Chatzipantazi V, Markou F, Tsakris A (2013) Outbreak caused by an ertapenem-resistant, CTX-M-15-producing Klebsiella pneumoniae sequence type 101 clone carrying an OmpK36 porin variant. J Clin Microbiol 51: 3176-3182.

15. Martínez-Martínez L, Conejo MC, Pascual A, HernándezAllés S, Ballesta S, Ramírez de Arellano-Ramos E, Benedí VJ, Perea EJ (2000) Activities of imipenem and cephalosporins against clonally related strains of Escherichia coli hyperproducing chromosomal $\beta$-lactamase and showing altered porin profiles. Antimicrob Agents Chemother 44: 2534-2536.

\section{Corresponding author}

Hugo Edgardo Villar, MD

Department of Clinical Bacteriology

Ladislao Martínez 43, Martínez (B1640EYA) Prov. de Buenos

Aires, Argentina

Phone: +541148985300

Email: hugo.villar@laboratoriohidalgo.com

Conflict of interests: No conflict of interests is declared. 